(2) Open Access Full Text Article

\title{
A colon-specific prodrug of metoclopramide ameliorates colitis in an experimental rat model
}

This article was published in the following Dove Medical Press journal:

Drug Design, Development and Therapy

\section{Yejin Yang* \\ Wooseong Kim* \\ Dayoon Kim \\ Seongkeun Jeong \\ Jin-Wook Yoo \\ Yunjin Jung}

College of Pharmacy, Pusan

National University, Busan 609-735,

South Korea

*These authors contributed equally to this work
Correspondence: Yunjin Jung

College of Pharmacy, Pusan National

University, Busan 609-735, South Korea

Tel +82 05I 5102527

Fax +82 05I 5136754

Email jungy@pusan.ac.kr
Background: We examined whether metoclopramide (MCP), a modulator of dopamine and serotonin receptors, alleviated colitis and had synergistic effects when coadministered with 5-aminosalicylic acid (5-ASA) in an experimental model of colitis.

Methods: MCP azo-linked to 5-ASA (5-[4-chloro-2-\{2-(diethylamino)ethylcarbamoyl\}1-methoxyphenyl]azosalicylic acid, MCP-azo-ASA) was synthesized, where 5-ASA was used as a colon-targeting carrier and an anti-colitic agent, and the ability of MCP-azo-ASA to target the colon in vitro and in vivo was evaluated.

Results: Our results indicate that MCP-azo-ASA was cleaved to MCP and 5-ASA in the cecal contents, but not in the contents of the small intestine. Oral gavage with equimolar concentrations of MCP-azo-ASA and sulfasalazine (SSZ, a colon-specific prodrug of 5-ASA widely used clinically) demonstrated that the two prodrugs delivered comparable amounts of 5-ASA to the cecum. MCP was barely detected in the blood after oral gavage with MCP-azo-ASA. In a rat model of 2,4-dinitrobenzene sulfonic acid hydrate (DNBS)-induced colitis, MCP-azo-ASA alleviated colonic damage in a dose-dependent manner. Moreover, MCP-azo-ASA reduced the concentrations of inflammatory mediators in the inflamed colon. At low equimolar doses, MCP-azo-ASA, but not SSZ, resulted in significant anti-colitic effects, which indicates that MCP has anti-colitic activity. MCP-azo-ASA had anti-colitic effects equal to those of SSZ at high equimolar doses. Conclusion: Thus, our results indicate that MCP-azo-ASA is a colon-specific prodrug of MCP. Targeted delivery of MCP to the colon ameliorated DNBS-induced colitis in rats, and we did not observe any synergistic effects of MCP after co-delivery with 5-ASA.

Keywords: metoclopramide, 5-aminosalicylic acid, colon-specific prodrug, colitis, serotonin, dopamine

\section{Introduction}

The enteric nervous system (ENS) of the gastrointestinal (GI) tract is the second nervous system in our body, and regulates the function of the GI system. The ENS innervates the lining of the entire GI tract from the esophagus to the anus, and does not only work independently, but also interacts with the central nervous system (CNS) through the autonomic nervous system and hormonal signaling pathways. ${ }^{1,2}$ The ENS-CNS communication, described as the gut-brain axis, plays an important role in the physiology of the GI tract, and a dysfunction of this axis causes disorders of the GI system. Stresses of different origins alter the functioning of the gut-brain axis and are associated with GI disorders, including inflammatory bowel disease (IBD), irritable bowel syndrome, and other functional GI diseases. ${ }^{1,5,6}$ Therefore, the gut-brain axis plays an important role in the pathophysiology of GI disorders, and understanding the role of 
this axis can provide a rationale for the development of new drugs and repositioning of the currently available drugs for the management of various GI disorders. Neurotransmitters such as serotonin (5-HT), norepinephrine, and dopamine ${ }^{7-9}$ play an important role in controlling the gut-brain axis, and many drugs can be used to modify the physiological actions of these neurotransmitters. ${ }^{7}$

IBD is an idiopathic disease caused by an impaired immune response to the host's intestinal microflora. The two major types of IBD are ulcerative colitis (UC), which is limited to the colonic mucosa, and Crohn's disease (CD), which can affect any segment of the GI tract from the mouth to the anus. ${ }^{10,11}$ Patients with IBD have abnormal levels of catecholamines and 5-HT, which are involved in regulating and controlling GI physiology, immunity, and microbiome. ${ }^{12-14}$ Several studies have shown that exogenous manipulation of these neurotransmitters is beneficial in experimental models of colitis. ${ }^{15-17}$ In fact, 5-HT3 receptors promote colonic inflammation while 5-HT4 receptors protect against colonic inflammation. ${ }^{18,19}$ Moreover, a D1- and D2-like dopamine receptor antagonist elicits anti-colitic activity by suppressing innate and adaptive immune responses. ${ }^{16}$

Metoclopramide (MCP) is mainly used for gastric and esophageal disorders, and for treating and preventing nausea, vomiting, and delayed emptying of the stomach. ${ }^{20}$ On the basis of its safety and efficacy, MCP has been listed as an essential medicine by the World Health Organization. MCP exerts its pharmacologic effects by acting as a dopamine receptor $2\left(\mathrm{D}_{2}\right)$, a $5-\mathrm{HT}_{3}$ receptor antagonist, and a $5-\mathrm{HT}_{4}$ receptor agonist. ${ }^{20,21}$ Recent studies have demonstrated that these effects of MCP on these receptors may be beneficial for ameliorating colitis through suppressing inflammation and reducing ulcers. ${ }^{16,18,19}$

To determine whether MCP can be used against colitis, we synthesized MCP that was azo-linked to 5-aminosalicylic acid (5-ASA) for targeted delivery to the colon, where 5-ASA was used as a colon-targeting and an anti-inflammatory agent. 5-ASA is an active ingredient of sulfasalazine (SSZ, a colonspecific prodrug of 5-ASA) currently used clinically for the treatment of IBD. ${ }^{22} \mathrm{MCP}$ azo-linked to 5-ASA (5-[4-chloro2-\{2-(diethylamino)ethylcarbamoyl $\}-1-$ methoxyphenyl] azosalicylic acid, MCP-azo-ASA) was expected to be colonspecific, thereby co-delivering MCP and 5-ASA to the large intestine. We administered two doses of MCP-azo-ASA by oral gavage to examine the anti-colitic effects of MCP alone and the synergistic effects of MCP and 5-ASA. Further, we monitored the systemic absorption of MCP after oral gavage with MCP-azo-ASA to examine the possibility of side effects.

\section{Materials and methods Materials}

MCP, salicylic acid, sodium nitrite $\left(\mathrm{NaNO}_{2}\right)$, sulfamic acid, and 5-ASA were purchased from Tokyo Kasei Kogyo Co. (Tokyo, Japan). SSZ and 2,4-dinitrobenzenesulfonic acid hydrate (DNBS) were purchased from Sigma Chemical Co. Inc. (St Louis, MO, USA). Reaction solvents were obtained from Junsei Chemical Co. (Tokyo, Japan). An ELISA kit was obtained from R\&D Systems (Minneapolis, MN, USA). All other chemicals used were reagent-grade commercially available products.

\section{Synthesis of MCP-azo-ASA}

We added $1.5 \mathrm{mmol}$ of $\mathrm{NaNO}_{2}$ to $1 \mathrm{mmol}$ of MCP dissolved in $3 \mathrm{~mL}$ of pre-chilled $18 \%$ hydrochloric acid, followed by stirring for 1 hour at $4{ }^{\circ} \mathrm{C}$ before the addition of sulfamic acid $(0.5 \mathrm{mmol})$. The resulting solution was added to salicylic acid ( $2 \mathrm{mmol}$ ) dissolved in $1 \mathrm{M} \mathrm{NaOH}(5 \mathrm{~mL})$ and then stirred at $4^{\circ} \mathrm{C}$ for 1 hour, followed by reaction at room temperature for 6 hours. The $\mathrm{pH}$ was adjusted to $9-10$ during the reaction. The precipitate was filtered and washed three times with diethyl ether/acetone (1:1) followed by drying in a vacuum oven. The infrared (IR) spectra were recorded on a Varian FT (Fourier transform)-IR spectrophotometer (Varian, Palo Alto, CA, USA). We obtained the proton nuclear magnetic resonance ( $\left.{ }^{1} \mathrm{H}-\mathrm{NMR}\right)$ imaging spectra by using a Varian AS 500 spectrometer, and the chemical shifts were in ppm downfield from tetramethylsilane. The yield of MCP-azo-ASA was $74 \%$; the melting point (mp) was $132^{\circ} \mathrm{C}-137^{\circ} \mathrm{C}$; IR (Nujol mull), $v_{\max }\left(\mathrm{cm}^{-1}\right): 1,627(\mathrm{C}=\mathrm{O}, \mathrm{CONH}), 1,580(\mathrm{C}=\mathrm{O}, \mathrm{COOH})$; and ${ }^{1} \mathrm{H}-\mathrm{NMR}$ (DMSO- $d 6$ ): $\delta=1.16$ (t, $6 \mathrm{H}, J=6.4 \mathrm{~Hz}$ ), 2.40-2.49 (m, 6H, overlapped with DMSO- $d 6$ signals), $3.12(\mathrm{~m}, 2 \mathrm{H})$, $3.62(\mathrm{~s}, 1 \mathrm{H}), 3.88$ (s, 3H), 6.71 (d, 1H, $J=8.4 \mathrm{~Hz}), 7.21$ $(\mathrm{s}, 1 \mathrm{H}), 7.75\left(\mathrm{dd}, J_{1}=8.4 \mathrm{~Hz}, J_{2}=2.4 \mathrm{~Hz}\right), 7.87(\mathrm{~s}, 1 \mathrm{H}), 8.13$ (d, $1 \mathrm{H}, J=2.4 \mathrm{~Hz}), 8.53(\mathrm{~s}, 1 \mathrm{H}), 9.94(\mathrm{~s}, 1 \mathrm{H})$.

\section{Apparent partition coefficient and chemical stability}

We added 1-octanol pre-saturated with isotonic phosphate buffer $(10 \mathrm{~mL}), \mathrm{pH} 6.8$, to a solution of MCP-azo-ASA $(1 \mathrm{mM}, 10 \mathrm{~mL})$ in an isotonic phosphate buffer pre-saturated with 1-octanol, $\mathrm{pH}$ 7.4. The mixture was shaken for 12 hours, and then left for phase separation at room temperature for 3 hours. The concentration of MCP-azo-ASA in the aqueous phase was determined using an ultraviolet (UV) spectrophotometer. The apparent partition coefficients (PCs) were calculated by using the equation $\left(\mathrm{C}_{\mathrm{o}}-\mathrm{C}_{\mathrm{w}}\right) / \mathrm{C}_{\mathrm{w}}$, where $\mathrm{C}_{\mathrm{o}}$ and 
$\mathrm{C}_{\mathrm{w}}$ represent the initial and equilibrium concentrations of the drug, respectively, in the aqueous phase. ${ }^{23}$ We performed the same experiment using MCP.

MCP-azo-ASA was placed in a hydrochloric acid buffer, $\mathrm{pH} 1.2$, or in an isotonic phosphate buffer, $\mathrm{pH} 6.8(500 \mu \mathrm{M}$, USP XXIII), and was incubated at $37^{\circ} \mathrm{C}$ for 10 hours. At a predetermined time interval, a $20 \mu \mathrm{L}$ aliquot of each solution was removed, and the concentrations of the drugs were analyzed using high-performance liquid chromatography (HPLC).

\section{HPLC analysis}

The HPLC system consisted of a model 306 pump, a 151 variable UV detector, and a model 234 autoinjector from Gilson (Middleton, WI, USA). We used a Symmetry R18 column $(250 \times 4.6 \mathrm{~mm}, 5 \mu \mathrm{m}$; Waters, Milford, MA, USA) with a guard column $(20 \times 4.6 \mathrm{~mm}$; Waters $)$. The samples prepared from each experiment were filtered using a $0.45 \mu \mathrm{m}$ membrane filter. HPLC analysis was conducted at a flow rate of $1 \mathrm{~mL} / \mathrm{min}$ using mobile phases consisting of acetonitrile and $3.0 \mathrm{mM} \mathrm{pH} 3.0$ phosphate buffer $(2: 8, \mathrm{v} / \mathrm{v})$ for MCP and acetonitrile, and $5.0 \mathrm{mM} \mathrm{pH} 7.4$ phosphate buffer $(2: 8, \mathrm{v} / \mathrm{v})$ for 5-ASA. The eluate was monitored at $323 \mathrm{~nm}$ (for 5-ASA) and $275 \mathrm{~nm}$ (for MCP) using a UV detector measuring the absorption with a sensitivity of 0.01 absorbance units full scale. The retention times of 5-ASA and MCP were 7.5 and 9.4 minutes, respectively.

\section{Conversion of MCP-azo-ASA to MCP in the GI tract}

A male Sprague Dawley (SD) rat weighing 250-260 g was euthanized using $\mathrm{CO}_{2}$, and a midline incision was made. The contents of the proximal small intestine, distal small intestine, and cecum were collected separately, and were suspended in an isotonic phosphate buffer, $\mathrm{pH} 6.8$, to prepare a $20 \%$ suspension. The cecal contents were collected under nitrogen in a nitrogen bag (Sigma). MCP-azo-ASA or SSZ in isotonic phosphate buffer, $\mathrm{pH} 6.8(0.5 \mathrm{~mL}, 1 \mathrm{mM})$, was added to the $20 \%(\mathrm{w} / \mathrm{v})$ suspension $(0.5 \mathrm{~mL})$ in microcentrifuge tubes, and the tubes were incubated at $37^{\circ} \mathrm{C}$ under nitrogen (for the cecal contents). The samples were centrifuged at $10,000 \times g$ for 5 minutes at specific time intervals. We added $0.9 \mathrm{~mL}$ of methanol to $0.1 \mathrm{~mL}$ of the supernatants; subsequently, the samples were vortexed and centrifuged at $20,000 \times g$ at $4^{\circ} \mathrm{C}$ for 10 minutes. The concentrations of 5-ASA and MCP in the supernatants were determined using HPLC.

\section{Rats}

Seven-week-old male SD rats were purchased from Samtako Bio Korea (Kyeong-gi-do, South Korea) and housed in the animal care facility at Pusan National University, Busan, South Korea. The SD rats were housed in the University's animal facility under controlled temperature, humidity, and dark/light cycle conditions. The animal protocol used in this study was reviewed and approved by the Pusan National University Institutional Animal Care and Use Committee (Approval Number: PNU-2017-1525, Approval date: 2017-04-19) for ethical procedures and scientific care. Animals were treated according to the National Institutes of Health guide for the Care and Use of Laboratory Animals.

\section{Induction of colitis}

Colitis was induced using DNBS according to the method for colitis induction using 2,4,6-trinitrobenzenesulfonic acid. ${ }^{24}$ Briefly, before the induction of colitis, the rats (250-260 g) were starved for 24 hours, but had free access to water. The rats were lightly anesthetized with isoflurane. A rubber cannula (OD, $2 \mathrm{~mm}$ ) was inserted rectally into the colon such that the tip was $8 \mathrm{~cm}$ proximal to the anus, approximately at the splenic flexure. DNBS dissolved in $50 \%(\mathrm{v} / \mathrm{v})$ aqueous ethanol was instilled into the colon via the rubber cannula (35 mg/0.35 mL/rat).

\section{Anti-colitic effects of drugs}

To evaluate the anti-colitic effects of drugs, each drug was suspended in $1 \mathrm{~mL}$ of PBS (pH 7.4). Rats were divided into six groups and treated as follows: group 1 (normal group): oral gavage with $1 \mathrm{~mL}$ PBS; group 2 (colitis group): oral gavage with $1 \mathrm{~mL}$ of PBS; group 3 (L-MCP-azo-ASA group): oral gavage with MCP-azo-ASA (6.7 mg/ $\mathrm{kg})$; group 4 (H-MCP-azo-ASA group): oral gavage with MCP-azo-ASA (33.8 $\mathrm{mg} / \mathrm{kg}$ ); group 5 (L-SSZ group): oral gavage with $\mathrm{SSZ}$ (6 mg/kg, equivalent to $6.7 \mathrm{mg} / \mathrm{kg}$ of MCP-azo-ASA); group 6 (H-SSZ group): oral gavage with SSZ (30 mg/kg, equivalent to $33.8 \mathrm{mg} / \mathrm{kg}$ of MCP-azo-ASA). SSZ or an equimolar concentration of MCP-azo-ASA was administered orally to rats once a day 72 hours after induction of colitis, and the rats were euthanized after receiving the treatment for 7 days. A gross colonic damage score (CDS) was calculated according to previously established criteria. ${ }^{24,25}$ The modified scoring system is as follows: 0 , normal appearance; 1 , localized hyperemia but no ulcer; 2 , linear ulcers without significant inflammation; 3, a 2-4 cm site of inflammation and ulceration; 4 , serosal adhesion to other organs and a $2-4 \mathrm{~cm}$ 
site of inflammation and ulceration; 5, stricture, serosal adhesion involving several bowel loops, and a $<4 \mathrm{~cm}$ site of inflammation and ulceration. Four independent observers blinded to the treatment assessed the CDS. Myeloperoxidase (MPO) activity was measured in the distal colon $(4 \mathrm{~cm})$ as described previously. ${ }^{24}$ One unit of MPO activity was defined as that degrading $1 \mu \mathrm{mol}$ of peroxide per minute at $25^{\circ} \mathrm{C}$.

\section{Determination of drug concentrations in plasma}

Healthy male SD rats were starved for 24 hours but allowed free access to water. MCP $(22.4 \mathrm{mg} / \mathrm{kg})$ or an equimolar concentration of MCP-azo-ASA (33.8 mg/kg) in PBS $(1.0 \mathrm{~mL})$ was administered by oral gavage. Blood samples were collected from the tail veins at specific time intervals and centrifuged at $4,000 \times g$ for 10 minutes. To a $0.1 \mathrm{~mL}$ aliquot of separated plasma, we added $1 \mathrm{M} \mathrm{NaOH}(0.01 \mathrm{~mL})$ followed by ethyl acetate $(0.5 \mathrm{~mL})$ and centrifuged samples at $4,000 \times g$ for 5 minutes. The organic layers were isolated and evaporated, and then dissolved in the mobile phase $(0.1 \mathrm{~mL})$. The concentration of MCP was determined in a $20 \mu \mathrm{L}$ aliquot using HPLC.

\section{Immunoblot analysis}

To prepare tissue lysates of the inflamed distal colon, the tissues $(1 \mathrm{~g})$ were disrupted and homogenized in $3 \mathrm{~mL}$ of ice cold RIPA buffer (50 mM Tris- $\mathrm{HCl}$ [pH 7.4], 1 mM EDTA, $0.7 \% \mathrm{Na}$ deoxycholate, $1 \% \mathrm{NP}-40,150 \mathrm{mM} \mathrm{NaCl}, 0.3 \mu \mathrm{M}$ aprotinin, $1 \mu \mathrm{M}$ pepstatin, and $1 \mathrm{mM}$ phenylmethylsulfonyl fluoride). After incubation on ice for 30 minutes, the homogenates were centrifuged at $10,000 \times g$ at $4^{\circ} \mathrm{C}$ for 10 minutes. Protein concentrations in the supernatants were determined using the bicinchoninic acid method. Tissue extracts were electrophoretically separated using 10\% sodium dodecyl polyacrylamide gel electrophoresis gels. We measured the concentrations of cyclooxygenase 2 (COX-2) and inducible nitric oxide synthase (iNOS) proteins in the tissue homogenates using a monoclonal anti-COX-2 antibody and an anti-iNOS (NOS-2) antibody (Santa Cruz Biotechnology, Santa Cruz, CA, USA) at a dilution of 1:1,000. Goat anti-mouse IgG-horseradish peroxidase (HRP) antibody (Abfrontier, Seoul, South Korea) and donkey anti-rabbit IgG-HRP antibody (Santa Cruz Biotechnology) were used as secondary antibodies, at dilutions of 1:2,000. The signals were visualized using the SuperSignal chemiluminescence substrate (Pierce, Rockford, IL, USA). The experiments were performed in triplicate, and the absorbance values were normalized with those obtained using antibodies to $\beta$-actin
(Santa Cruz Biotechnology). Blot images were detected with Bio-Rad ChemiDoc Touch Imaging System (Bio-Rad, Hercules, CA, USA).

\section{Cytokine-induced neutrophil chemoattractant- 3 immunoassay}

To measure the concentrations of cytokine-induced neutrophil chemoattractant-3 (CINC-3) in the inflamed tissues, we homogenized the distal colon in a potassium phosphate buffer, $\mathrm{pH}$ 6, and centrifuged homogenates at 10,000× $g$ at $4^{\circ} \mathrm{C}$ for 10 minutes. An appropriate volume of the supernatant was analyzed using a CINC-3 ELISA kit (R\&D Systems).

\section{Data analysis}

The results are expressed as mean \pm standard error of the mean (SEM). ANOVA followed by Tukey's honest significant difference test or the Mann-Whitney $U$-test (for CDS) was used to test for statistical differences between the data. Differences with $\alpha$ or $P<0.05$ were considered significant.

\section{Results}

\section{Synthesis of MCP-azo-ASA}

The process of synthesis of MCP-azo-ASA is shown in Figure 1. Formation of the final product was verified using IR and ${ }^{1} \mathrm{H}-\mathrm{NMR}$ spectroscopy. While the carbonyl stretching band ascribed to the carboxylic acid in 5-ASA was observed at $1,627 \mathrm{~cm}^{-1}$ in the IR spectra, the IR spectra showed a shift in this band to $1,580 \mathrm{~cm}^{-1}$ in MCP-azo-ASA, because of the zwitterionic property of MCP-azo-ASA acquired by the formation of an azo bond between 5-ASA and MCP, which was confirmed by the disappearance of the $\mathrm{N}-\mathrm{H}$ stretching bands (detected at 3,399 $\mathrm{cm}^{-1}$ and $3,320 \mathrm{~cm}^{-1}$ ) ascribed to aniline in MCP. The results of ${ }^{1} \mathrm{H}-\mathrm{NMR}$ indicate that the signals of the aliphatic protons attached to the tertiary amine in MCP-azo-ASA were shifted downfield compared to those of MCP. In addition, ${ }^{1} \mathrm{H}$-NMR signals originating from the protons in the aromatic rings of 5-ASA and MCP were detected between 6.7 and $8.2 \mathrm{ppm}$ in MCP-azo-ASA. IR and ${ }^{1} \mathrm{H}-\mathrm{NMR}$ spectra of 5-ASA, MCP, and MCP-azoASA are shown in Figure S1.

\section{MCP-azo-ASA is a colon-specific prodrug of MCP}

We evaluated whether MCP-azo-ASA was a colon-specific prodrug of MCP in vitro and in vivo. The PCs of MCP-azoASA and MCP were measured in an n-octanol (pH 6.8) buffer system, and chemical modification of MCP decreased the PC of MCP from 2.614 to 0.296 , which indicated that 


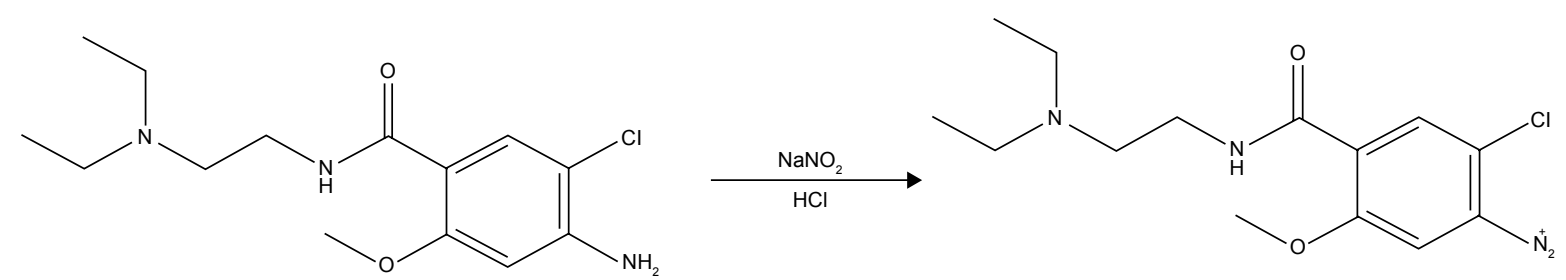

Metoclopramide
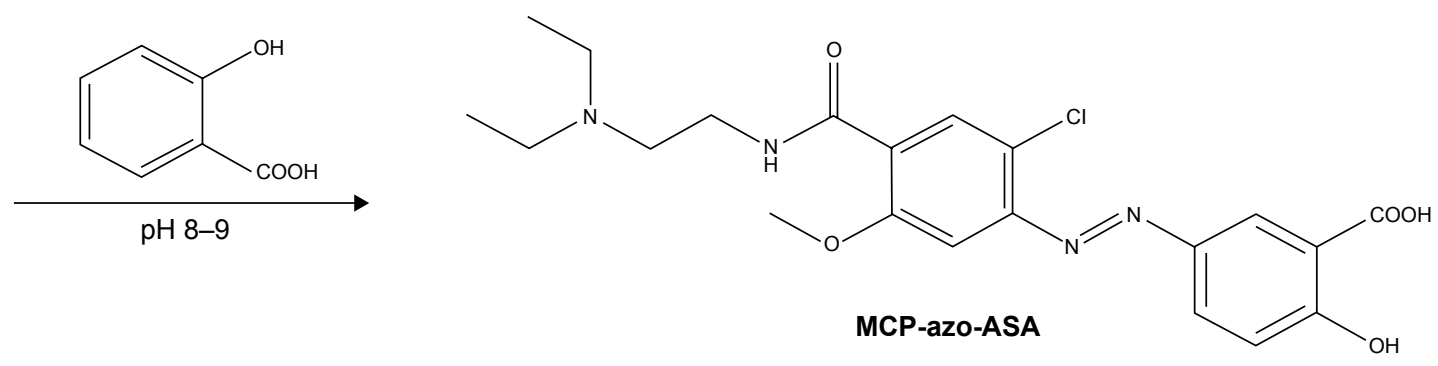

Figure I Synthesis of 5-(4-chloro-2-[2-\{diethylamino\}ethylcarbamoyl]-I-methoxyphenyl)azosalicylic acid (MCP azo-linked to 5-ASA, MCP-azo-ASA). Abbreviation: MCP-azo-ASA, metoclopramide azo-linked to 5-aminosalicylic acid.

MCP-azo-ASA was more hydrophilic. Incubation of MCPazo-ASA with contents of the small intestine and buffers of $\mathrm{pH} 1.2$ and $\mathrm{pH} 6.8$ showed no presence of either 5-ASA or MCP in the media, and no change in the concentrations of MCP-azo-ASA. On the contrary, MCP-azo-ASA was cleaved to release $\mathrm{MCP}$ in the cecal contents, and the percent release of MCP was $\sim 34.6 \%$ at 4 hours and $49.7 \%$ at 8 hours (Figure 2A). The rate of cleavage of MCP-azo-ASA in the cecal contents was compared with that of SSZ, a colonspecific prodrug of 5-ASA with an azo-linkage. The rate of cleavage of MCP-azo-ASA was similar to that of SSZ (Figure 2B). The role of microbial enzymes in the cecal activation of MCP-azo-ASA was determined by autoclaving the cecal contents as reported previously, ${ }^{23}$ followed by the addition of MCP-azo-ASA. MCP was not detected in the cecal contents up to 24 hours. To determine targeted delivery of MCP-azo-ASA to the colon, we orally administered MCP-azo-ASA to the rats and measured the concentrations of 5-ASA in the cecum at specific time intervals. The same experiment was performed using SSZ, and the results of both experiments were compared. The concentrations of 5-ASA released from MCP-azo-ASA were comparable to those released from SSZ in the cecum at 2, 4, and 6 hours after oral gavage with MCP-azo-ASA and SSZ (Figure 2C). On the other hand, the concentrations of MCP released from MCP-azo-ASA were similar to those of 5-ASA. The cecal concentrations of MCP were $\sim 1.1$ (at 2 hours), 3.4 (at 4 hours), and 2.8 (at 6 hours) $\mathrm{mmol} / \mathrm{kg}$. Targeted delivery of a drug to the colon reduces the side effects of the drug by decreasing its systemic absorption. ${ }^{26}$ We determined the systemic absorption of MCP by measuring the concentrations of MCP in the blood after gavage with MCP-azo-ASA. For comparison, the same experiment was performed using MCP. Although millimolar concentrations of MCP accumulated in the cecum after oral gavage with MCP-azo-ASA, MCP was barely detected in the blood. On the other hand, oral gavage with MCP resulted in MCP concentrations of up to $\sim 1.84 \mu \mathrm{M}$ in the blood (Figure 2D). Our results demonstrate that MCPazo-ASA effectively delivers MCP to the large intestine without significant systemic absorption of MCP, thereby increasing the therapeutic availability in the colon, and likely avoiding the systemic effects of MCP.

\section{MCP-azo-ASA ameliorates DNBS- induced colitis in rats}

To determine the efficacy of MCP-azo-ASA against colitis, we administered MCP-azo-ASA via oral gavage once a day 3 days after the induction of colitis by DNBS. The same experiment was performed using SSZ as a positive control. The abovementioned experiment was performed using two doses. The CDS and MPO activities were determined after treatment with the prodrugs for 7 days. As shown in Figure 3A, the normal segment of the colon showed no damage, but the distal colon of the group treated with DNBS showed severe damage with inflammatory edema, destruction of the mucosa, intraluminal stricture, and serosal adhesion to other organs. While a low dose of SSZ (6 mg/ $\mathrm{kg})$ had no significant effect on the CDS, a dose of MCP-azo-ASA 
A

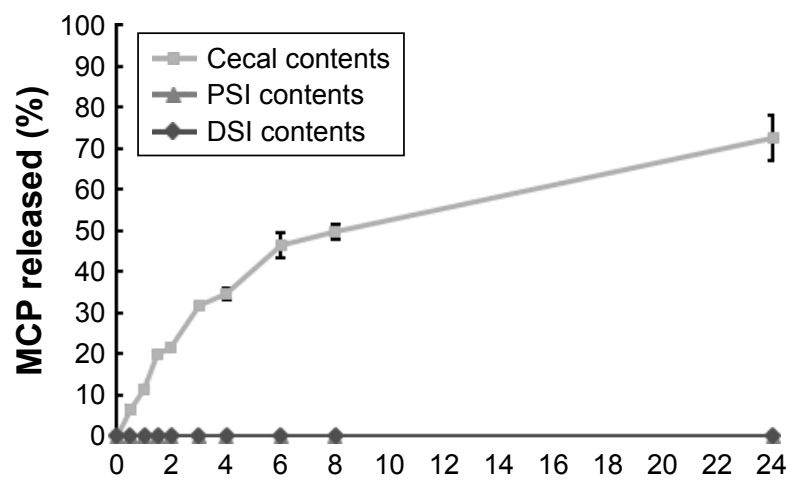

C

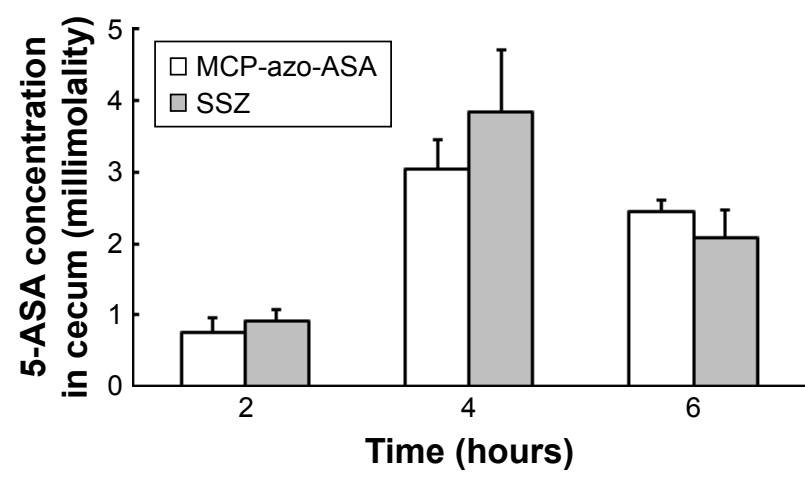

B

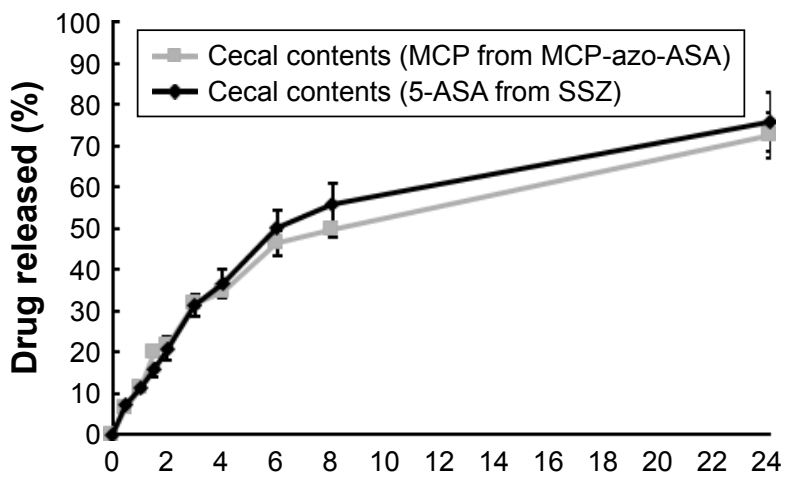

D

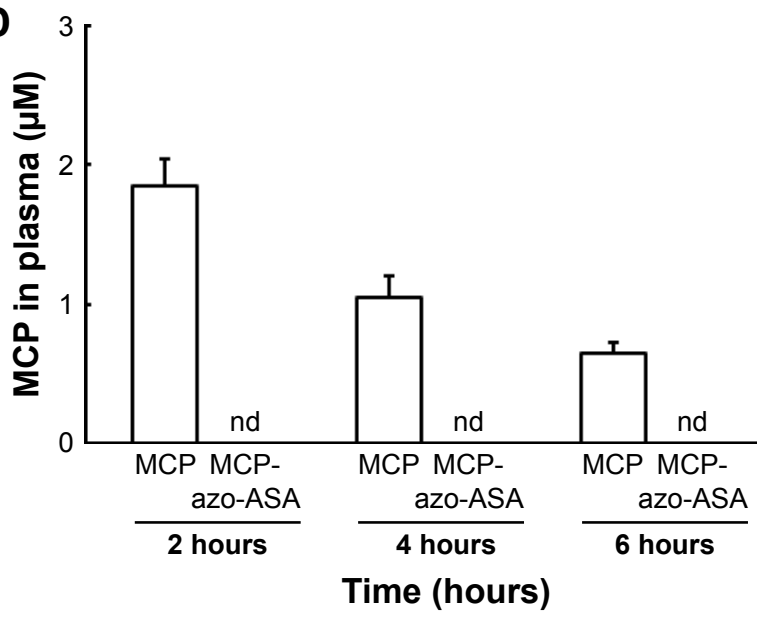

Figure 2 MCP-azo-ASA delivers MCP to the colon and limits the systemic absorption of MCP.

Notes: (A) MCP-azo-ASA ( $1 \mathrm{mg} / \mathrm{mL}$ ) was added to the contents of the cecum and the small intestine suspended in PBS (I0\%, $\mathrm{pH} 6.8)$. The concentrations of MCP in the samples were determined at specific time intervals using HPLC. (B) The experiment (performed in cecal contents) described in (A) was repeated with an equimolar concentration of SSZ, and the results of both experiments were compared. Male SD rats ( $250-260 \mathrm{~g})$ were starved for 24 hours but allowed free access to water. (C) SSZ $(30 \mathrm{mg} / \mathrm{kg})$ or an equimolar concentration of MCP-azo-ASA $(33.8 \mathrm{mg} / \mathrm{kg}$ ) suspended in PBS $(\mathrm{pH} 7.4, \mathrm{I} \mathrm{mL})$ was administered to rats by oral gavage. The rats were euthanized 2,4 , and 6 hours after oral gavage, and a midline incision was made to obtain the cecum. The concentrations of 5 -ASA in the cecal contents were analyzed by HPLC. (D) MCP $(22.4 \mathrm{mg} / \mathrm{kg})$ or an equimolar concentration of MCP-azo-ASA $(33.8 \mathrm{mg} / \mathrm{kg}$ ) was administered to rats by oral gavage, and blood samples were collected at predetermined intervals from the tail veins. The concentrations of MCP in the blood were analyzed using HPLC. The data in $\mathbf{A}-\mathbf{D}$ are shown as mean \pm SEM ( $n=5$ ).

Abbreviations: 5-ASA, 5-aminosalicylic acid; MCP, metoclopramide; MCP-azo-ASA, metoclopramide azo-linked to 5-aminosalicylic acid; SD, Sprague Dawley; SSZ, sulfasalazine; PSI, proximal small intestine; DSI, distal small intestine; nd, not detectable; HPLC, high-performance liquid chromatography; SEM, standard error of the mean.

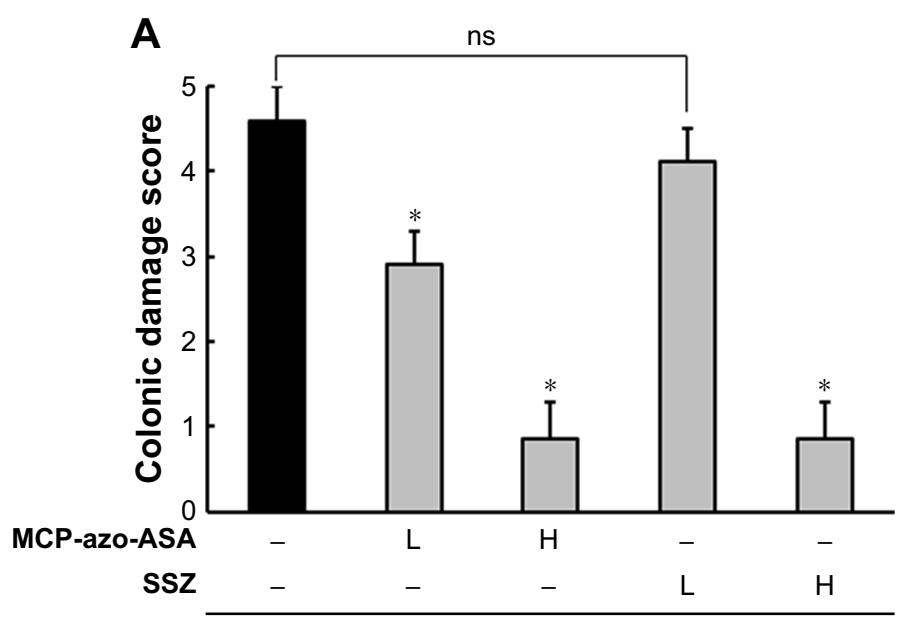

DNBS

Figure 3 (Continued) 
B
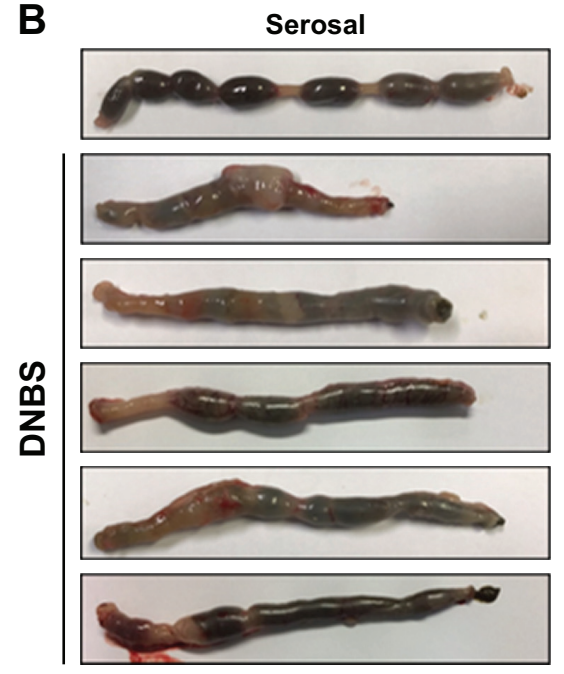

C

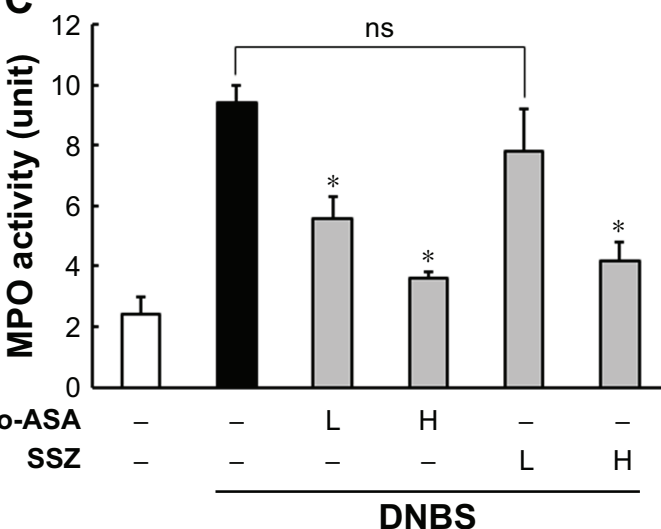

Luminal
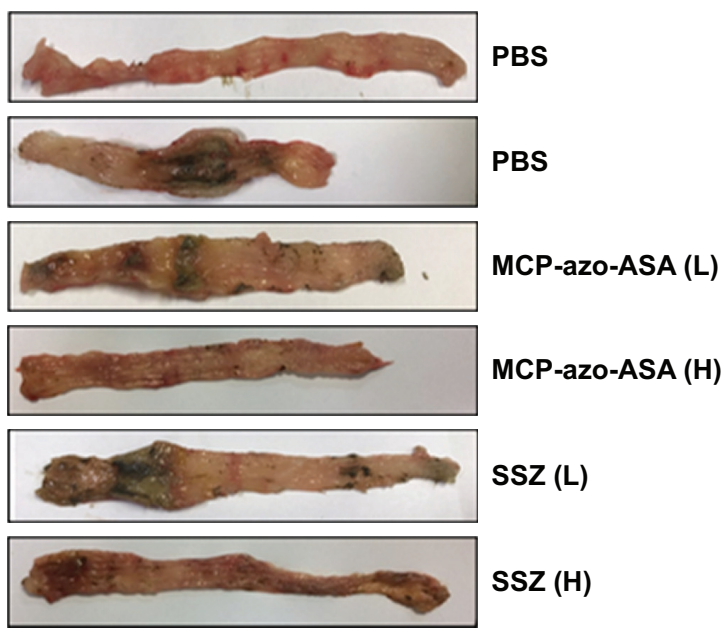

SSZ (L)

SSZ (H)

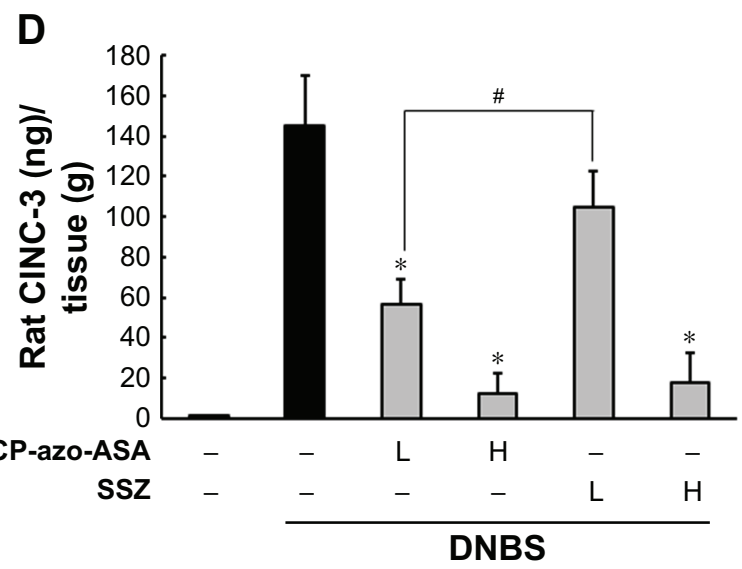

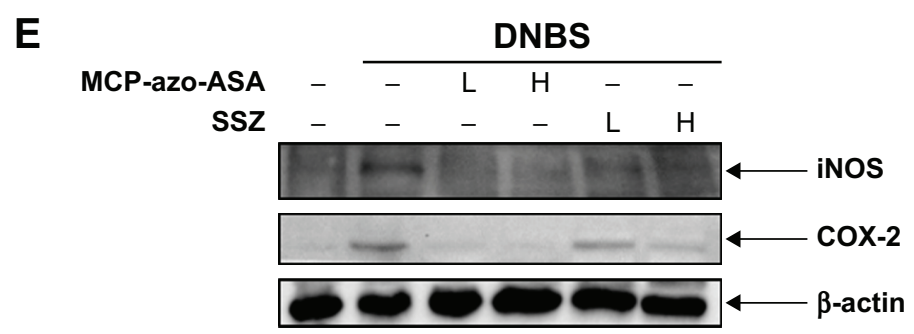

Figure 3 MCP-azo-ASA ameliorates DNBS-induced colitis in rats.

Notes: Male SD rats (250-260 g) were starved for 24 hours but allowed free access to water. For low-dose groups (represented as L), MCP-azo-ASA (6.7 mg/kg) or SSZ $(6 \mathrm{mg} / \mathrm{kg})$ suspended in PBS (I mL, pH 7.4) was administered to rats with DNBS-induced colitis by oral gavage once a day, and the rats were euthanized 7 days after treatment with the drugs. The same experiment was repeated with MCP-azo-ASA (33.8 mg/kg) and SSZ (30 mg/kg) for the high-dose group (represented as H). (A) CDS was assigned for each group. (B) The luminal and serosal sides of distal colons were photographed. (C) MPO activities were measured using the distal colon (4 cm). (D) The concentrations of CINC-3 were determined in the inflamed distal colons. (E) The concentrations of COX-2 and iNOS in the inflamed distal colons were measured using Western blot analysis. The results of Western blotting are representative of three independent experiments. The data in $\mathbf{A}, \mathbf{C}$, and $\mathbf{D}$ are shown as mean $\pm S E M(n=5)$. $* P<0.05$ vs $D N B S$ control (in $\mathbf{A},{ }^{*} \alpha<0.05$ vs colitis control), ${ }^{*} P<0.05$.

Abbreviations: CDS, colonic damage score; CINC-3, cytokine-induced neutrophil chemoattractant-3; COX-2, cyclooxygenase 2; DNBS, 2,4-dinitrobenzene sulfonic acid hydrate; iNOS, inducible nitric oxide synthase; MCP-azo-ASA, metoclopramide azo-linked to 5-aminosalicylic acid; MPO, myeloperoxidase; SD, Sprague Dawley; SSZ, sulfasalazine; SEM, standard error of the mean; ns, not significant.

equimolar to that of SSZ resulted in a significant improvement in the CDS. At high doses $(30 \mathrm{mg} / \mathrm{kg} \mathrm{SSZ}$ and an equimolar concentration of MCP-azo-ASA), both prodrugs resulted in a remarkable improvement in the CDS, and the efficacy of MCP-azo-ASA was as high as that of SSZ.
The images of the distal colons are shown in Figure 3B. The results of MPO activity were similar to those of CDS, in that irrespective of its dose, MCP-azo-ASA significantly reduced the MPO activity in the inflamed colon, whereas the MPO activity was reduced only at high doses of SSZ (Figure 3C). 
In addition, we evaluated the molecular indices of inflammation by determining the concentrations of inflammatory mediators such as CINC-3, COX-2, and iNOS in the inflamed colon. A low dose of MCP-azo-ASA, but not of $\mathrm{SSZ}$, significantly reduced the concentrations of inflammatory mediators CINC-3 (Figure 3D) and COX-2 and iNOS (Figure 3E). Furthermore, the reduction in the concentrations of the inflammatory mediators observed when a high dose of MCP-azo-ASA was used was similar to that observed when a high dose of SSZ was used. These results suggest that MCP had an ability to alleviate colitis and did not exert additive anti-colitic effects after co-delivery with 5-ASA.

\section{MCP is marginally effective in alleviating DNBS-induced colitis in rats}

MCP delivered specifically to the large intestine resulted in significant anti-colitic effects. Thus, we examined whether conventional delivery of MCP was effective for treatment of colitis in rats. Anti-colitic effects were evaluated after oral gavage with MCP (equimolar to a high dose of MCPazo-ASA). Administration of MCP improved the CDS (Figure 4A) and reduced the activity of MPO (Figure 4B) in the inflamed colon, although the reduction in MPO activity was not statistically significant. The images of the distal colons are shown in Figure S2. MCP also attenuated the concentrations of the inflammatory mediators CINC-3 (Figure 4C), iNOS, and COX-2 (Figure 4D). Overall, MCP equimolar to a high dose of MCP-azo-ASA was no more effective in alleviating rat colitis than a low dose of MCPazo-ASA, as shown in Figure 3A-E.

\section{Discussion}

We synthesized MCP azo-linked to 5-ASA, MCP-azo-ASA, and evaluated whether this was a colon-specific prodrug of MCP. MCP-azo-ASA delivered MCP and 5-ASA specifically to the large intestine with no systemic absorption of
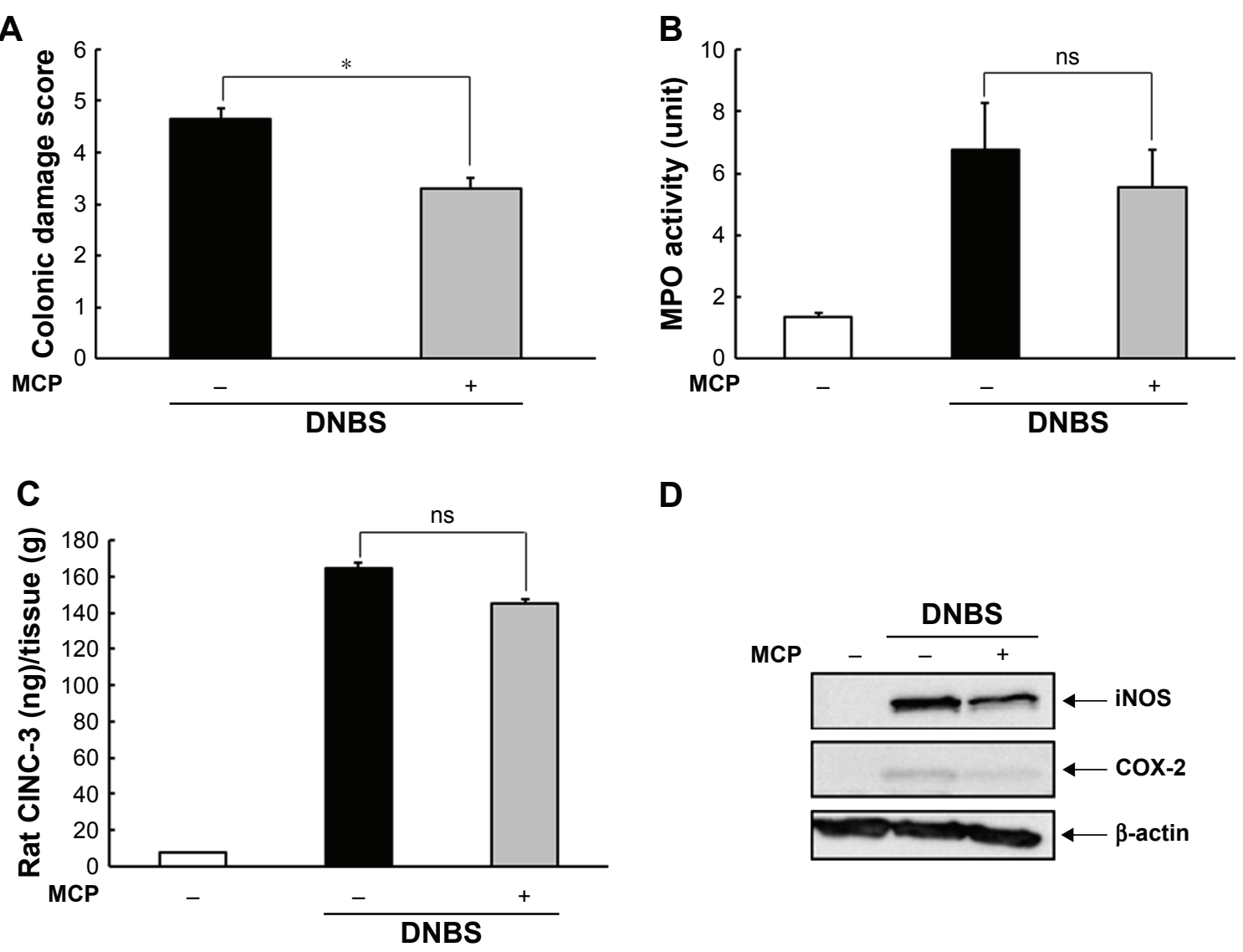

D

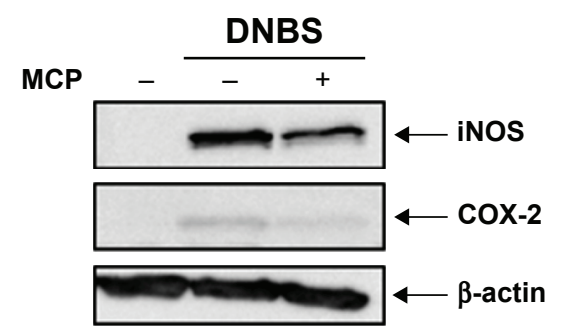

Figure $4 \mathrm{MCP}$ is marginally effective in ameliorating DNBS-induced colitis in rats.

Notes: Male SD rats (250-260 g) were starved for 24 hours but allowed free access to water. MCP ( $22.4 \mathrm{mg} / \mathrm{kg}$, equimolar to $33.8 \mathrm{mg} / \mathrm{kg}$ MCP-azo-ASA) suspended in PBS ( $\mathrm{mL}$, pH 7.4) was administered to rats with DNBS-induced colitis by oral gavage once a day, and the rats were euthanized 7 days after treatment with the drug. (A) CDS was assigned for each group. The data are shown as mean $\pm \operatorname{SEM}(n=5), * \alpha<0.05$. (B) MPO activities were measured using the distal colon ( $4 \mathrm{~cm})$. The concentrations of CINC-3 (C), COX-2 and iNOS (D) were determined in the inflamed distal colons. The Western blotting results are representative of three independent experiments. The data in B and $\mathbf{C}$ are shown as mean $\pm \operatorname{SEM}(\mathrm{n}=5), * \mathrm{P}<0.05$.

Abbreviations: CDS, colonic damage score; CINC-3, cytokine-induced neutrophil chemoattractant-3; COX-2, cyclooxygenase 2; DNBS, 2,4-dinitrobenzene sulfonic acid hydrate; iNOS, inducible nitric oxide synthase; MCP, metoclopramide; MPO, myeloperoxidase; SD, Sprague Dawley; SEM, standard error of the mean; ns, not significant. 
MCP, and MCP delivered to the large intestine resulted in significant anti-colitic activity, whereas no significant additive anti-colitic effects were observed after co-administration of 5-ASA and MCP.

Our in vitro and in vivo data suggest that MCP-azo-ASA can be effectively delivered to the large intestine and be converted to MCP and 5-ASA. Our results demonstrate that oral gavage with MCP-azo-ASA and SSZ resulted in the release of similar amounts of 5-ASA from both prodrugs in the cecum, and thus the efficiency of the delivery of MCPazo-ASA was comparable to that of SSZ. Our results indicate that the rate of cleavage of MCP-azo-ASA in the cecum was not significantly different from that of SSZ, and thus the cecal 5-ASA concentration was directly associated with the efficiency of delivery. The prodrug was thought to be activated by microbial enzymes such as azoreductases, since the conversion of MCP-azo-ASA to MCP did not take place in the autoclaved cecal contents, where microbial enzymes are inactivated. ${ }^{23}$

While MCP was barely detected in the blood after oral gavage with MCP-azo-ASA, oral gavage with MCP resulted in blood concentrations of up to $1.84 \mu \mathrm{M}$ of $\mathrm{MCP}$, which suggested that systemic absorption was largely limited because of targeted delivery of MCP to the colon. This finding is important in repositioning MCP as an anti-colitic agent. In addition to the toxicological effects of MCP, such as movement disorders, depressed mood, seizure, and jaundice, all pharmacological effects manifested by the systemic absorption of MCP could be considered side effects after repositioning of the drug. ${ }^{27}$ In addition, a large percentage of patients with IBD receiving long-term SSZ therapy experience moderate to serious side effects, such as agranulocytosis and hypospermia, because of the systemic absorption of sulfapyridine generated from SSZ in the large intestine. ${ }^{28}$ Therefore, MCP-azo-ASA may have toxicological advantages over SSZ.

MCP alone had the ability to suppress the colonic inflammation induced by DNBS. Our results demonstrate that at a low dose, MCP-azo-ASA, but not SSZ, had significant anti-colitic effects. Thus, the anti-colitic effects of MCP-azoASA were primarily governed by MCP rather than 5-ASA, given that SSZ is a colon-specific prodrug consisting of antiinflammatory 5-ASA and sulfapyridine "inert" against colitis. The anti-colitic effects of low dose of MCP-azo-ASA were not sufficient to result in a statistical significance between the two groups treated with MCP-azo-ASA and SSZ. Moreover, we did not observe any significant additive anti-colitic effects after co-delivery of 5-ASA and MCP. While SSZ was effective against colitis at a high dose, which indicated that 5-ASA exerted anti-colitic effects, a high dose of MCP-azo-ASA did not result in enhanced anti-colitic effects, which suggests that there are no synergistic effects between 5-ASA and MCP.

The observation of no synergistic effect between MCP and 5-ASA (delivered to the colon) may be because of the therapeutic feature of SSZ in the large intestine. Recent studies indicate that SSZ acts as a mutual prodrug because SSZ coexists with 5-ASA in the inflamed large intestine until complete conversion to 5-ASA, and SSZ itself exerts anti-colitic effects through the inhibition of nuclear factor kappa B (NF-кB). ${ }^{24,29}$ Thus, SSZ may exert anti-colitic effects through both 5-ASA and sulfasalazine. In addition, the conditions for animal experiments may not be suitable to show subtle therapeutic differences between MCP-azo-ASA and SSZ. Animal experiments with elaborate experimental conditions such as an optimized drug dose, drug treatment period, and dose of DNBS for induction of colitis may be required to determine the therapeutic advantage of MCPazo-ASA over sulfasalazine.

Our results demonstrate that MCP (equimolar to a high dose of MCP-azo-ASA) seems less effective in ameliorating rat colitis than MCP-azo-ASA at a low dose, which suggests that MCP-azo-ASA may have more potent anti-colitic effects than MCP. This is likely ascribed to the targeted delivery of MCP by MCP-azo-ASA to the colon, considering that colon-targeted delivery of a drug tends to increase potency of the drug for the treatment of IBD. ${ }^{26}$ Oral gavage with MCP-azo-ASA achieved MCP concentrations in the large intestine that are sufficient to act on dopamine and serotonin receptors, and thus, colonic MCP was very likely to modulate the activities of the receptors in the inflamed colons, likely resulting in beneficial effects on colitis. ${ }^{16,18,19}$ Furthermore, the anti-colitic effects of colonic MCP may be exerted by additional mechanisms, because millimolar concentrations of MCP, which were never achieved by conventional MCP as shown in Figure 2D, were afforded at the target site, thus acting on other targets, such as NF- $\mathrm{KB} \cdot{ }^{30}$ Further studies are required to determine the mechanism underlying the anticolitic effects of MCP delivered specifically to the colon.

In conclusion, MCP-azo-ASA is a potential colon-specific prodrug of MCP. MCP delivered specifically to the colon alleviated colonic inflammation and did not have significant additive anti-colitic effects after co-delivery with 5-ASA.

\section{Acknowledgments}

This research was supported by the Basic Science Research Program, through the National Research Foundation of Korea (NRF), funded by the Ministry of Education (2018R1D1A3B07045694). 


\section{Disclosure}

The authors report no conflicts of interest in this work.

\section{References}

1. Bonaz BL, Bernstein CN. Brain-gut interactions in inflammatory bowel disease. Gastroenterology. 2013;144(1):36-49.

2. Al Omran Y, Aziz Q. The brain-gut axis in health and disease. Adv Exp Med Biol. 2014;817:135-153.

3. Ohman L, Simrén M. Pathogenesis of IBS: role of inflammation, immunity and neuroimmune interactions. Nat Rev Gastroenterol Hepatol. 2010;7(3):163-173.

4. Rhee SH, Pothoulakis C, Mayer EA. Principles and clinical implications of the brain-gut-enteric microbiota axis. Nat Rev Gastroenterol Hepatol. 2009;6(5):306-314.

5. Camilleri M. Management of the irritable bowel syndrome. Gastroenterology. 2001;120(3):652-668.

6. Brzozowski B, Mazur-Bialy A, Pajdo R, et al. Mechanisms by which stress affects the experimental and clinical inflammatory bowel disease (IBD): role of brain-gut axis. Curr Neuropharmacol. 2016;14(8): 892-900.

7. Mittal R, Debs LH, Patel AP, et al. Neurotransmitters: the critical modulators regulating gut-brain axis. J Cell Physiol. 2017;232(9): 2359-2372.

8. Crowell MD, Wessinger SB. 5-HT and the brain-gut axis: opportunities for pharmacologic intervention. Expert Opin Investig Drugs. 2007;16(6):761-765.

9. Mayer EA, Tillisch K, Bradesi S. Review article: modulation of the brain-gut axis as a therapeutic approach in gastrointestinal disease. Aliment Pharmacol Ther. 2006;24(6):919-933.

10. Ko JK, Auyeung KK. Inflammatory bowel disease: etiology, pathogenesis and current therapy. Curr Pharm Des. 2014;20(7):1082-1096.

11. Scharl M, Rogler G. Inflammatory bowel disease pathogenesis: what is new? Curr Opin Gastroenterol. 2012;28(4):301-309.

12. Coates MD, Tekin I, Vrana KE, Mawe GM. Review article: the many potential roles of intestinal serotonin (5-hydroxytryptamine, 5-HT) signalling in inflammatory bowel disease. Aliment Pharmacol Ther. 2017;46(6):569-580.

13. Tolstanova G, Deng X, Ahluwalia A, et al. Role of dopamine and D2 dopamine receptor in the pathogenesis of inflammatory bowel disease. Dig Dis Sci. 2015;60(10):2963-2975.

14. Magro F, Vieira-Coelho MA, Fraga S, et al. Impaired synthesis or cellular storage of norepinephrine, dopamine, and 5-hydroxytryptamine in human inflammatory bowel disease. Dig Dis Sci. 2002;47(1):216-224.

15. Levin AD, van den Brink GR. Selective inhibition of mucosal serotonin as treatment for IBD? Gut. 2014;63(6):866-867.
16. Kawano M, Takagi R, Kaneko A, Matsushita S. Berberine is a dopamine D1- and D2-like receptor antagonist and ameliorates experimentally induced colitis by suppressing innate and adaptive immune responses. J Neuroimmunol. 2015;289:43-55.

17. Straub RH, Grum F, Strauch U, et al. Anti-inflammatory role of sympathetic nerves in chronic intestinal inflammation. Gut. 2008;57(7): 911-921.

18. Spohn SN, Bianco F, Scott RB, et al. Protective actions of epithelial 5 -hydroxytryptamine 4 receptors in normal and inflamed colon. Gastroenterology. 2016;151(5):933-944.

19. Utsumi D, Matsumoto K, Amagase K, Horie S, Kato S. 5-HT3 receptors promote colonic inflammation via activation of substance $\mathrm{P} /$ neurokinin-1 receptors in dextran sulphate sodium-induced murine colitis. Br J Pharmacol. 2016;173(11):1835-1849.

20. Albibi R, Mccallum RW. Metoclopramide: pharmacology and clinical application. Ann Intern Med. 1983;98(1):86-95.

21. Sanger GJ. Translating 5-HT receptor pharmacology. Neurogastroenterol Motil. 2009;21(12):1235-1238.

22. Peppercorn MA. Sulfasalazine. Pharmacology, clinical use, toxicity, and related new drug development. Ann Intern Med. 1984;101(3):377-386.

23. Kim H, Kong H, Choi B, et al. Metabolic and pharmacological properties of rutin, a dietary quercetin glycoside, for treatment of inflammatory bowel disease. Pharm Res. 2005;22(9):1499-1509.

24. Kim W, Nam J, Lee S, Jeong S, Jung Y. 5-Aminosalicylic Acid AzoLinked to Procainamide Acts as an Anticolitic Mutual Prodrug via Additive Inhibition of Nuclear Factor KappaB. Mol Pharm. 2016;13(6): 2126-2135.

25. Yano H, Hirayama F, Kamada M, Arima H, Uekama K. Colon-specific delivery of prednisolone-appended alpha-cyclodextrin conjugate: alleviation of systemic side effect after oral administration. J Control Release. 2002;79(1-3):103-112.

26. Jung Y, Kim YM. What should be considered on design of a colonspecific prodrug? Expert Opin Drug Deliv. 2010;7(2):245-258

27. Parkman HP, Mishra A, Jacobs M, et al. Clinical response and side effects of metoclopramide: associations with clinical, demographic, and pharmacogenetic parameters. J Clin Gastroenterol. 2012;46(6): 494-503.

28. Linares V, Alonso V, Domingo JL. Oxidative stress as a mechanism underlying sulfasalazine-induced toxicity. Expert Opin Drug Saf. 2011;10(2):253-263.

29. Gan HT, Chen YQ, Ouyang Q. Sulfasalazine inhibits activation of nuclear factor-kappaB in patients with ulcerative colitis. J Gastroenterol Hepatol. 2005;20(7):1016-1024.

30. Morissette G, Moreau E, C-Gaudreault R, Marceau F. N-substituted 4-aminobenzamides (procainamide analogs): an assessment of multiple cellular effects concerning ion trapping. Mol Pharmacol. 2005; 68(6):1576-1589. 


\section{Supplementary materials}

A

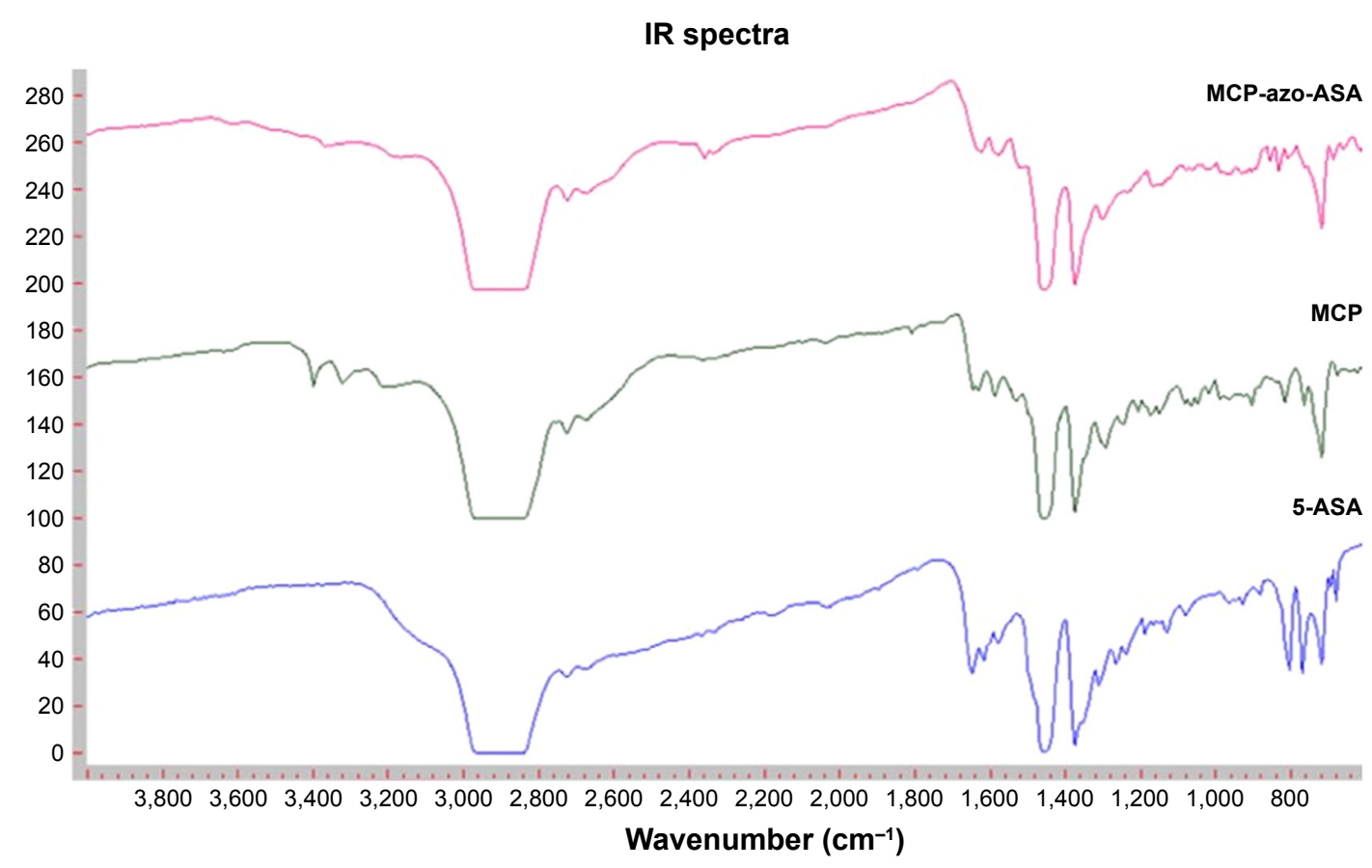

B

NMR spectra
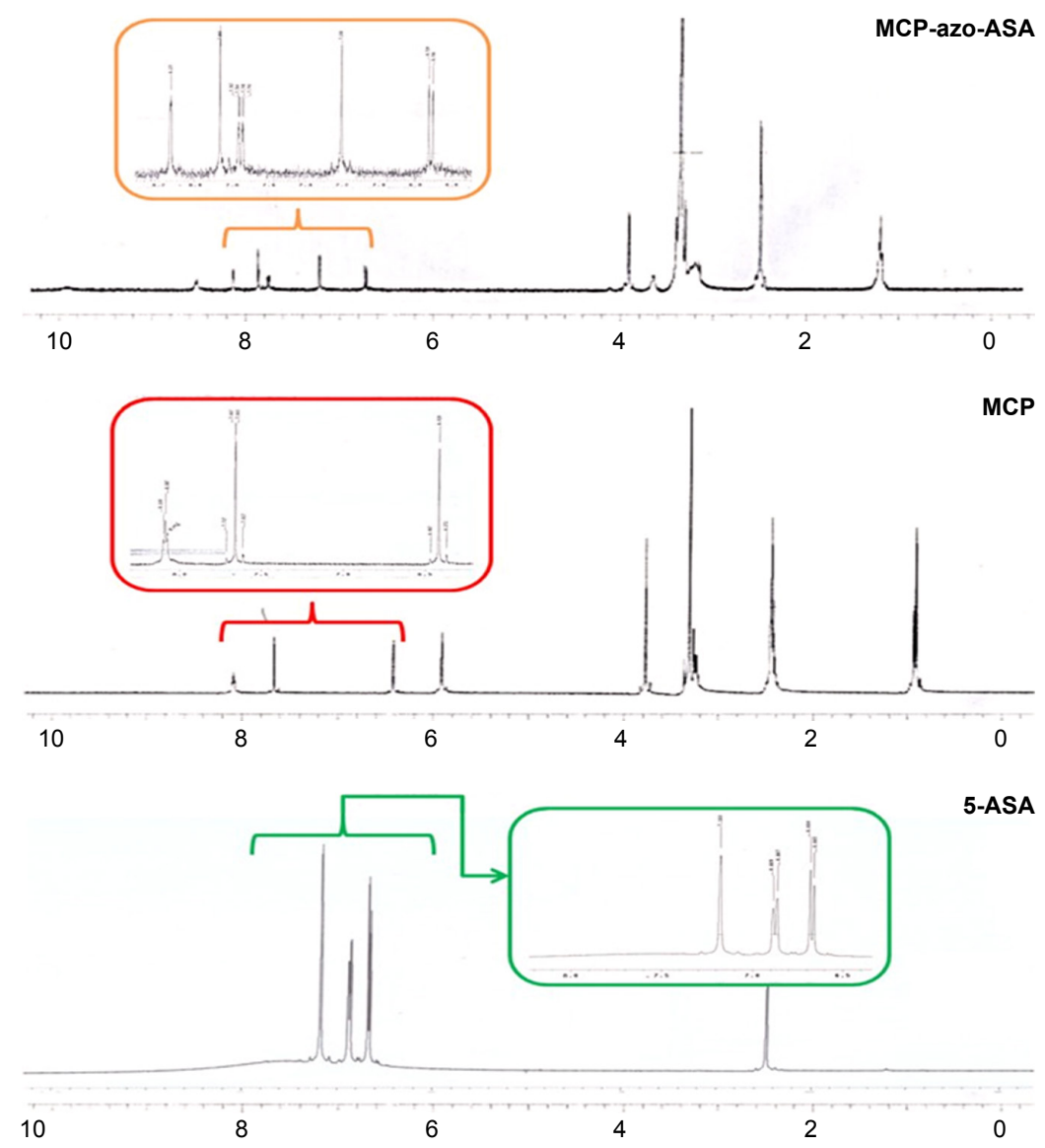

Figure SI IR (A) and 'H-NMR (B) spectra of 5-aminosalicylic acid (5-ASA), metoclopramide (MCP), and metoclopramide azo-linked to 5-aminoslicylic acid (MCP-azo-ASA). Abbreviations: IR, infrared; 'H-NMR, proton nuclear magnetic resonance. 

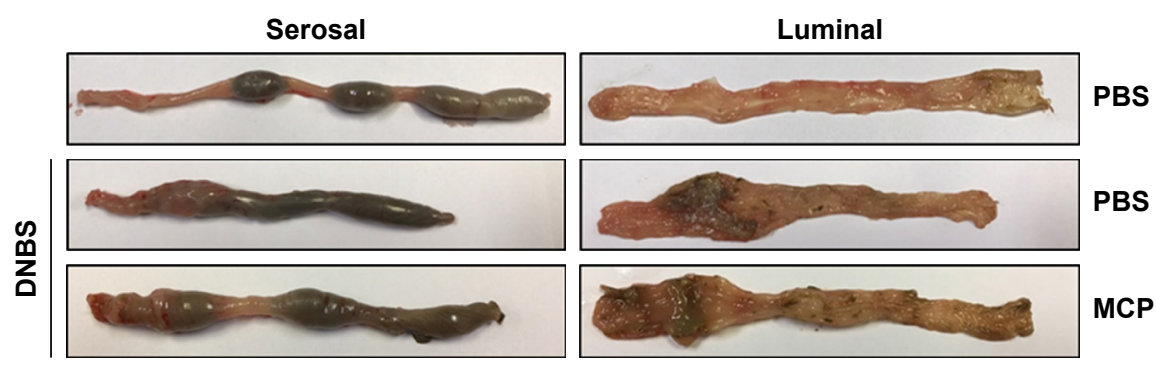

Figure S2 Photos of the luminal and serosal sides of distal colons.

Notes: Metoclopramide (MCP, $22.4 \mathrm{mg} / \mathrm{kg}$, equimolar to $33.8 \mathrm{mg} / \mathrm{kg}$ MCP-azo-ASA) was administered to rats with 2,4-dinitrobenzene sulfonic acid hydrate (DNBS)-induced colitis by oral gavage once a day, and the rats were euthanized 7 days after treatment with the drug. The luminal and serosal sides of distal colons were photographed. Abbreviation: MCP-azo-ASA, metoclopramide azo-linked to 5-aminosalicylic acid.

\section{Publish your work in this journal}

Drug Design, Development and Therapy is an international, peerreviewed open-access journal that spans the spectrum of drug design and development through to clinical applications. Clinical outcomes, patient safety, and programs for the development and effective, safe, and sustained use of medicines are the features of the journal, which has also been accepted for indexing on PubMed Central. The manuscript management system is completely online and includes a very quick and fair peer-review system, which is all easy to use. Visit http://www.dovepress.com/testimonials.php to read real quotes from published authors.

Submit your manuscript here: http://www.dovepress.com/drug-design-development-and-therapy-journal 